Y. C. Minh and E. F. van Dishoeck, eds.

\title{
Observations of Gas and Dust in Comets with the Infrared Space Observatory
}

\author{
J. Crovisier \\ Observatoire de Paris-Meudon, F-92195 Meudon, France
}

\begin{abstract}
The Infrared Space Observatory (ISO) offered us the opportunity to observe celestial bodies from 2.4 to $196 \mu \mathrm{m}$, which is of particular interest for comets. We present here spectroscopic observations with ISO of comets $\mathrm{C} / 1995 \mathrm{O} 1$ (Hale-Bopp) and 103P/Hartley 2. Fluorescence emissions of $\mathrm{H}_{2} \mathrm{O}, \mathrm{CO}_{2}$ and (for comet Hale-Bopp only) $\mathrm{CO}$ and $\mathrm{CH}_{4}$ are observed, yielding the production rates of these species. High-resolution spectra of water permit the study of the rotational and spin temperatures of this molecule. In comet Hale-Bopp, the thermal infrared region of the spectra shows specifically, in addition to continuum-like emission, the signatures of crystalline, $\mathrm{Mg}$-rich olivine (forsterite), and signs of crystalline pyroxenes, amorphous silicates and water ice. The presence of crystalline silicates is also suggested in the Jupiter-family comet P/Hartley 2.
\end{abstract}

\section{Introduction}

Waiting for the direct investigation of cometary nuclei by space missions, we can only study the chemical composition of these bodies by observing the material they are rejecting during periods of cometary activity.

Recently, the Infrared Space Observatory (ISO) (Kessler et al. 1996) gave us access to the full infrared range from 2.4 to $196 \mu \mathrm{m}$. This spectral range, which covers the domain of thermal emission, the fundamental vibrational bands of gas-phase molecules, and specific bands of dust and ice constituents, is thus fully adequate for an investigation of the chemical and physical properties of cometary material (Fig. 1).

ISO could observe in some detail the spectra of the exceptionally bright, long-period comet C/1995 O1 (Hale-Bopp) and of the Jupiter-family comet 103P/Hartley 2 (Table 1). This provides an interesting comparison, these two comets having presumably different formation histories. A summary of the ISO results on these two comets is presented here. ${ }^{1}$

\footnotetext{
${ }^{1}$ This paper concentrates on spectroscopic results. Other cometary programmes with ISO, aimed at imaging (with ISOCAM) and broad-band photometry (with ISOPHOT) of comet Hale-Bopp and short-period comets, will not be reported here.
} 
C/1995 01 (Hale-Bopp) 7 Oct. 1996

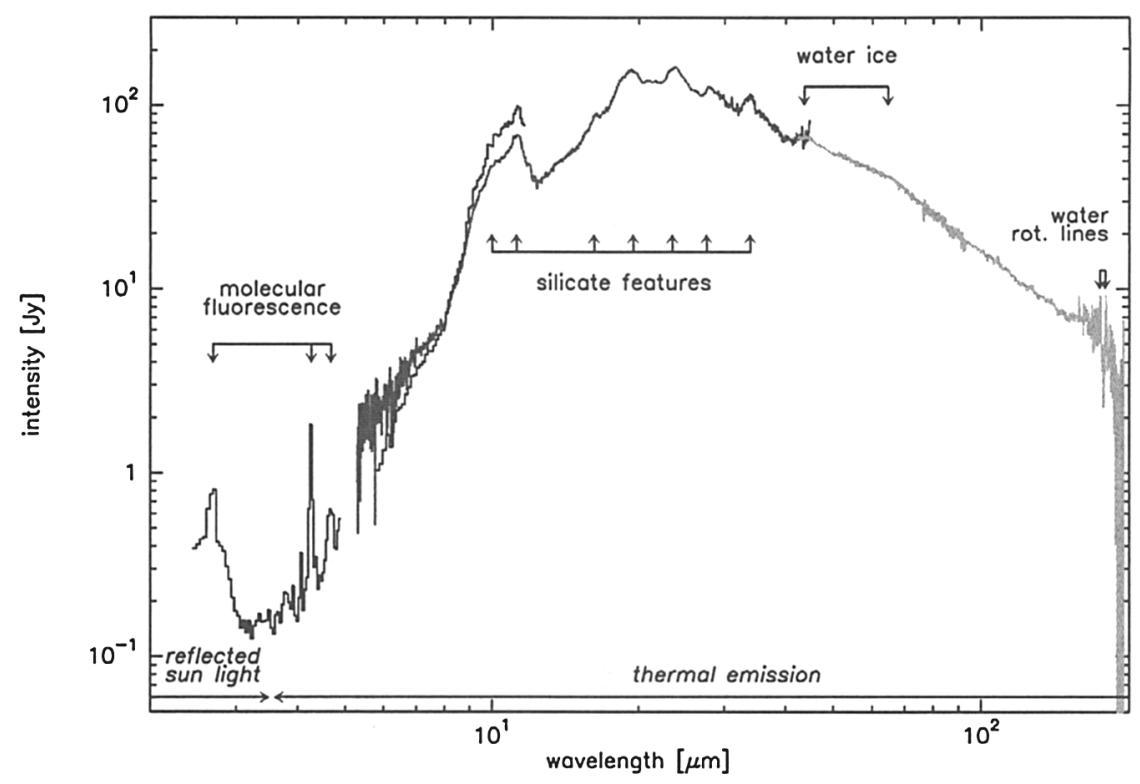

Figure 1. A composite spectrum of C/1995 O1 (Hale-Bopp) from 2.5 to $195 \mu \mathrm{m}$ obtained with the PHT-S (2.5-5 and 6-12 $\mu \mathrm{m})$, SWS $(7-45 \mu \mathrm{m})$ and LWS $(43-195 \mu \mathrm{m})$ instruments of ISO. The LWS spectrum has been arbitrarily rescaled in intensity to make the spectrum continuous.

\section{Observations}

Comet C/1995 O1 (Hale-Bopp) was observed by ISO as a target of opportunity (Crovisier et al. 1996, 1997a,b, 1999a, 2000; Lellouch et al. 1998). It is an exceptionally bright comet, presumably coming from the Oort cloud. Spectra were obtained pre-perihelion on April 1996 and September - October 1996, and post-perihelion on December 1997 and April 1998, spanning heliocentric distances $\left(r_{h}\right)$ between 2.8 and $4.9 \mathrm{AU}$. Unfortunately, this comet could not be observed around perihelion with ISO because of visibility constraints. At the largest heliocentric distances, only low-resolution spectra were obtained with the photometer (PHT-S; spectral range 2.5-5 and 6-12 $\mu \mathrm{m}$, resolution $\lambda / \Delta \lambda \approx 80$, field of view $\left.24^{\prime \prime} \times 24^{\prime \prime}\right)$. At the smallest $r_{h}$ 's, a high-resolution spectrum covering the full 2.4 to $196 \mu \mathrm{m}$ spectral range was obtained for the first time in a comet, using the short-wavelength spectrometer (SWS; $2.4-45 \mu \mathrm{m}$, resolution $\approx 1500$, field of view $14^{\prime \prime} \times 20^{\prime \prime}$ to $20^{\prime \prime} \times 33^{\prime \prime}$ ) and the long-wavelength spectrometer (LWS; $43-195 \mu \mathrm{m}$, resolution $\approx 200$, field of view $100^{\prime \prime}$ ).

Other spectroscopic programmes were devoted to short-period comets of the Jupiter-family, presumably originating from the Edgeworth-Kuiper belt. 22P/Kopff was observed on October-December 1996 with SWS and LWS. Due to the weakness of the object, which was then at $r_{h}=1.9 \mathrm{AU}$, only the $\nu_{3}$ ro- 
Table 1. Properties of the two bright comets observed spectroscopically with ISO.

\begin{tabular}{cc}
\hline $\mathrm{C} / 1995 \mathrm{O} 1$ (Hale-Bopp) & $103 \mathrm{P} /$ Hartley 2 \\
\hline long-period $(P \approx 4000$ years $)$ & Jupiter-family $(P=6.3$ years $)$ \\
from Oort Cloud & from Kuiper Belt \\
formed at $r_{h}<35 \mathrm{AU}$ & formed at $r_{h}>35 \mathrm{AU}$ \\
$Q_{\max }\left[\mathrm{H}_{2} \mathrm{O}\right]=10^{31} \mathrm{~s}^{-1}$ & $Q_{\max }\left[\mathrm{H}_{2} \mathrm{O}\right]=10^{28} \mathrm{~s}^{-1}$ \\
observed at $2.8<r_{h}<4.9 \mathrm{AU}$ & observed at $r_{h} \approx 1 \mathrm{AU}$ \\
\hline
\end{tabular}

Table 2. Molecular production rates derived from the SWS and PHT-S spectra.

\begin{tabular}{lccccc}
\hline Date & $\begin{array}{c}r_{h} \\
(\mathrm{AU})\end{array}$ & $\begin{array}{c}\mathrm{H}_{2} \mathrm{O} \\
\left(10^{28}\right.\end{array}$ & $\left.\begin{array}{c}\mathrm{CO} \\
\text { molec. }\end{array} \mathrm{s}^{-1}\right)$ & $\mathrm{CH}_{4}$ \\
\hline$C / 1995$ & O1 & (Hale-Bopp) & & & \\
$27 / 04 / 96$ & 4.58 & & 7.0 & 1.3 & \\
$26 / 09 / 96$ & 2.93 & 33.0 & 23.0 & 7.4 & 0.55 \\
$29 / 12 / 97$ & 3.89 & 2.8 & 15.0 & 3.5 & \\
$06 / 04 / 98$ & 4.90 & & 30.0 & 3.0 & \\
$103 P /$ Hartley 2 & & & & \\
$01 / 01 / 98$ & 1.04 & 1.2 & & 0.12 & \\
\hline
\end{tabular}

vibrational lines of water were detected, with SWS (Crovisier et al. 1999b). Comet 103P/Hartley 2 was observed close to its perihelion (at $r_{h}=1.04 \mathrm{AU}$ from Sun and 0.82 AU from Earth) on January 1998 with SWS, LWS, PHT-S and the CVF $(5-17 \mu \mathrm{m}, \lambda / \Delta \lambda \approx 41)$ of the imaging instrument ISOCAM (Colangeli et al. 1999b; Crovisier et al. 1999b, 2000).

\section{Results}

\subsection{Gas composition}

In comet Hale-Bopp, the vibrational bands of water, carbon dioxide, carbon monoxide and methane are detected in emission (Figures 2 and 5), as expected from molecular fluorescence models. Relative production rates $\mathrm{H}_{2} \mathrm{O}: \mathrm{CO}_{2}: \mathrm{CO}: \mathrm{CH}_{4}$ were observed to be $100: 20: 70: 1.5$ at $r=2.9$ AU. However, $\mathrm{H}_{2} \mathrm{O}: \mathrm{CO}_{2}: \mathrm{CO}$ strongly vary as a function of heliocentric distance (Table 2 ), as could be expected from the different volatilities of these species. For instance, the onset of sublimation of water is expected to occur around $r_{h}=4-5 \mathrm{AU}$. Therefore, one must be cautious when extrapolating the relative abundances of 

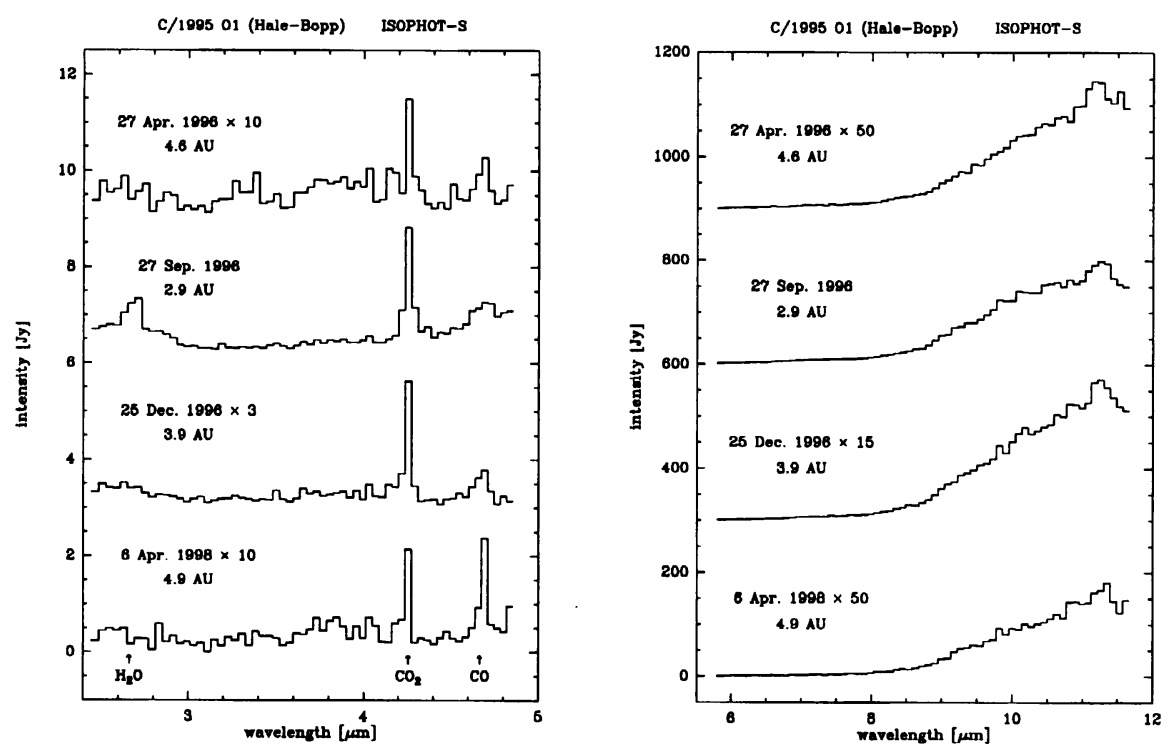

Figure 2. The 2.5-5 $\mu \mathrm{m}$ (left) and 6-12 $\mu \mathrm{m}$ (right) spectra of comet C/1995 O1 (Hale-Bopp) observed with ISOPHOT-S at four different dates, showing evolution with heliocentric distance. Note the different intensity scales (from: Crovisier et al. 1999a.)

cometary volatiles observed in the coma to the true composition of cometary ices in the nucleus.

In comet $\mathrm{P} / \mathrm{Hartley} 2$, the bands of $\mathrm{H}_{2} \mathrm{O}$ and $\mathrm{CO}_{2}$ at 2.7 and $4.3 \mu \mathrm{m}$ are detected with PHT-S (Colangeli et al. 1999b; Fig. 3), with CAM-CVF and with SWS (Crovisier et al. 1999b; Fig. 5). Relative production rates $\mathrm{CO}_{2}: \mathrm{H}_{2} \mathrm{O}=$ $10 \%$ are observed (Table 2). Therefore, $\mathrm{CO}_{2}$ is found to be a major cometary volatile in both comets, and appears to be the easiest gas species to detect at these wavelengths at large $r_{h}$. It must be noted that space-borne infrared spectroscopy is the only direct way to observe this molecule.

\subsection{Gas physical properties}

In comet Hale-Bopp, several rotational lines of $\mathrm{H}_{2} \mathrm{O}$ were observed with the LWS (Fig. 4). This is the first observation of rotational water lines in a comet. ${ }^{2}$ These lines are heavily thick and need to be interpreted using sophisticated radiative transfer-excitation models.

\footnotetext{
${ }^{2}$ Subsequently, the $1_{10}-1_{01}$ line of water was observed through heterodyne techniques by SWAS in comet C/1999 H1 (Lee) (Bergin et al. 1999).
} 


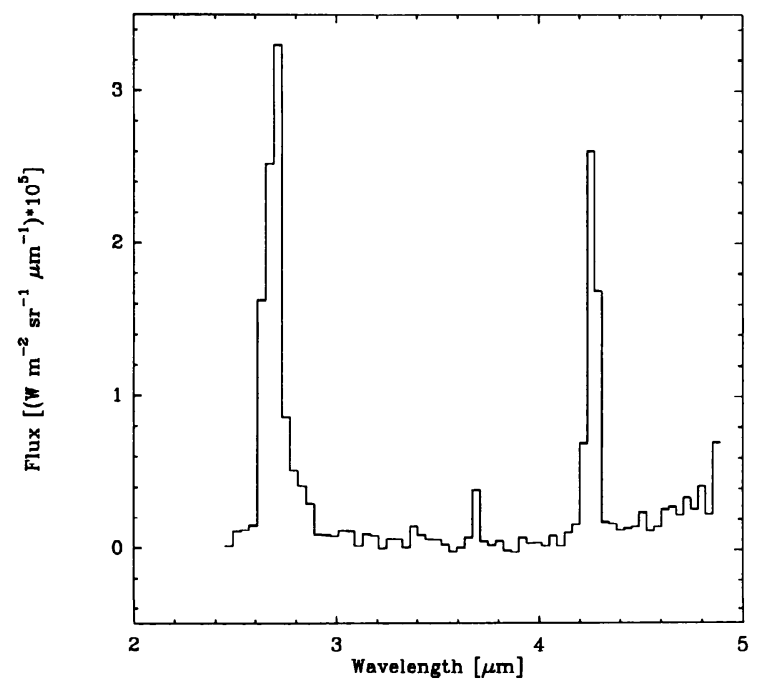

Figure 3. The 2.5-5 $\mu$ m spectrum of comet 103P/Hartley 2 obtained with ISOPHOT-S on 1 January 1998, showing the strong bands of water at $2.7 \mu \mathrm{m}$ and carbon dioxide at $4.65 \mu \mathrm{m}$ (from: Colangeli et al. $1999 \mathrm{~b})$.

The bands of $\mathrm{H}_{2} \mathrm{O}$ around $2.7 \mu \mathrm{m}\left(\nu_{1}, \nu_{3}\right.$ and hot bands) (Fig. 5) and $6 \mu \mathrm{m}$ $\left(\nu_{2}\right.$ band) were observed with SWS at high spectral resolution. In September - October 1996, the ro-vibrational lines of the $\nu_{3}$ band were observed with a high signal-to-noise ratio. This allows an accurate determination of the water rotational temperature $(28 \mathrm{~K})$ and of its ortho-to-para ratio $(2.45 \pm 0.10$, which significantly differs from the high temperature limit and corresponds to a spin temperature of $28 \mathrm{~K}$ ) (Table 3 ).

In comet $\mathrm{P} / \mathrm{Hartley} 2$, the $2.7 \mu \mathrm{m}$ band of $\mathrm{H}_{2} \mathrm{O}$ is also observed with a high signal-to-noise ratio (Fig. 5), which permits to evaluate the rotational temperature of water averaged within the field of view to $20 \mathrm{~K}$, and its ortho-to-para ratio to $\approx 2.7$, corresponding to a spin temperature $T_{\text {spin }} \approx 35 \mathrm{~K}$ (Table 3 ).

Table 3. Water rotational temperature $\left(T_{\text {rot }}\right)$, ortho-to-para ratio (OPR) and spin temperature $\left(T_{\text {spin }}\right)$ derived from the high-resolution spectra.

\begin{tabular}{lcccc}
\hline Comet & date & $\begin{array}{c}T_{\text {rot }} \\
(\mathrm{K})\end{array}$ & OPR & $\begin{array}{c}T_{\text {spin }} \\
(\mathrm{K})\end{array}$ \\
\hline C/Hale-Bopp & Sep. to Oct. 98 & 28.5 & $2.45 \pm 0.10$ & $28 \pm 2$ \\
103P/Hartley 2 & 1 Jan. 98 & $20.3 \pm 0.8$ & $2.76 \pm 0.08$ & $36 \pm 3$ \\
& 19 Jan. 98 & $16.5 \pm 1.6$ & $2.63 \pm 0.18$ & $33 \pm 5$ \\
\hline
\end{tabular}


C/1995 01 (Hale-Bopp) ISO/LWS 6 October 1996

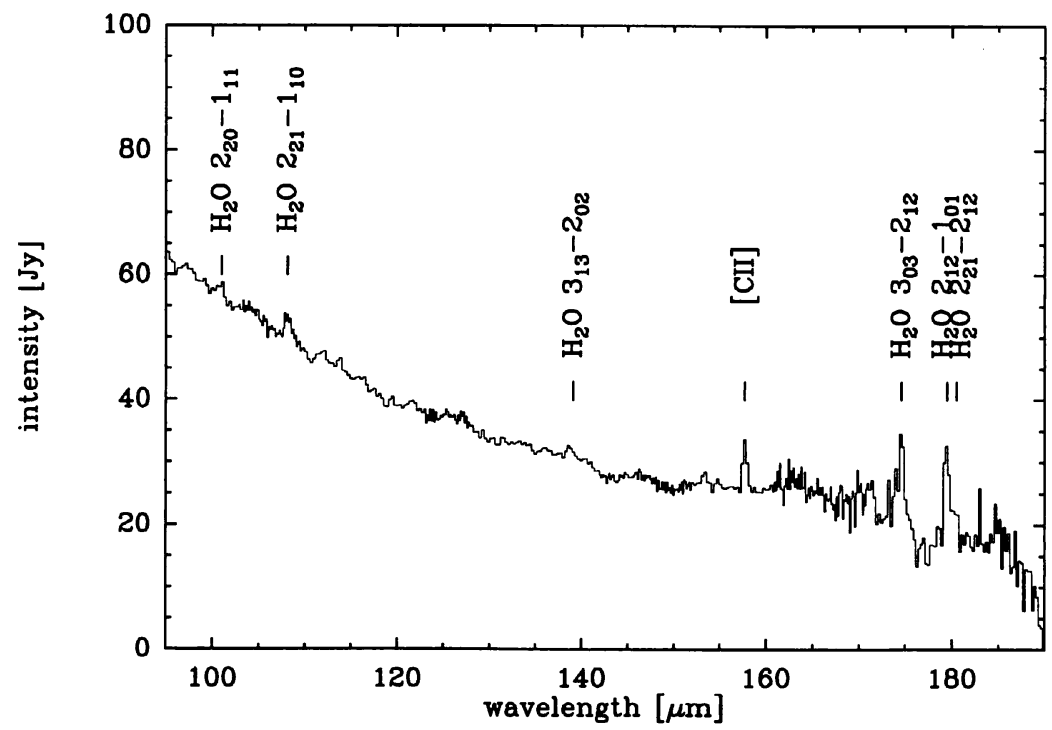

Figure 4. Several rotational lines of water observed with ISO-LWS in comet $\mathrm{C} / 1995 \mathrm{O} 1$ (Hale-Bopp). The Cir line is not from the comet; it comes from the background (from: Crovisier et al. 1999a).

The cold rotational temperatures are in agreement with hydrodynamical models of cometary atmospheres which predict cooling of cometary atmospheres during their expansion. The higher temperature in comet Hale-Bopp, despite its larger $r_{h}$, may be attributed to a more efficient thermalization of the photolysis products, due to its higher coma density.

The present spin temperature determinations, which rely on the analysis of lines of low optical depth for the whole $\nu_{3}$ water band, are believed to be more reliable than previous ones (from KAO observations of comets $1 \mathrm{P} / \mathrm{Halley}$ and $\mathrm{C} / 1986 \mathrm{P} 1 \mathrm{Wilson}$ ). Their meaning is still a debated issue, however (see Mumma et al. 1993 for a summary of previous measurements and a discussion of the problem). $T_{\text {spin }}$ could be the temperature of water at formation on the surface of cold grains, since gas-phase reactions are exothermal and would yield "hot" water. Alternatively, the ortho-to-para ratio could re-equilibrate within the nucleus, $T_{s p i n}$ being then the equilibrium temperature of nucleus ices. Finally, it could betray fractionation effects when water condenses or sublimates. These two last mechanisms require a laboratory investigation of ortho-to-para conversion mechanisms for water under cometary conditions.

\subsection{Icy grains and dust}

Beyond $6 \mu \mathrm{m}$, the spectrum of comet Hale-Bopp is dominated by dust thermal continuum emission, upon which broad emission features are superposed (Figures 1 and 6). In the September - October 1996 spectra, emission features at 45 and $65 \mu \mathrm{m}$ (Fig. 1) and a possible absorption at 2.9-3.2 $\mu \mathrm{m}$ are seen, charac- 


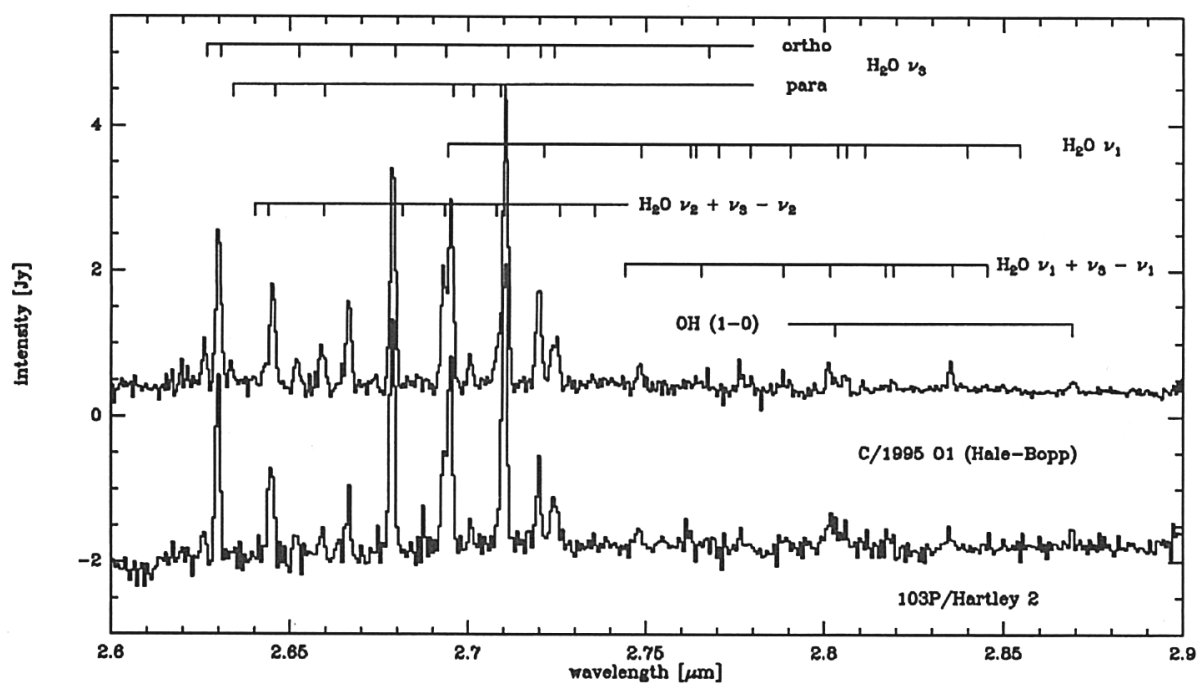

Figure 5. The region of the $\nu_{3}$ band of water observed with ISOSWS in comets C/1995 O1 (Hale-Bopp), average of 27 Sept. and 6 Oct. 1996 (top) and 103P/Hartley 2, 1st Jan. 1998 (bottom) (adapted from Crovisier et al. 1997a and 1999b).

teristic of crystalline water ice (Lellouch et al. 1998). This suggests that water ice grains were still present at $r=3 \mathrm{AU}$ in the coma, and that they could be a significant source of volatiles.

The wavelengths of the other prominent features closely match those of Mgrich crystalline olivine (forsterite $\mathrm{Mg}_{2} \mathrm{SiO}_{4}$ ) (Crovisier et al. 1997a). A detailed analysis of the spectrum, using laboratory spectra of various kinds of silicates (Crovisier et al. 2000), also reveals the presence of pyroxene and amorphous silicates. The ratio of crystalline to amorphous silicates is found to be of the order of $40 \%$ by mass. The continuum emission cannot be interpreted in terms of a single-temperature blackbody emission. Observations at different $r_{h}$ 's, before and after perihelion, do not show any significant evolution of the dust properties, except of course changes in the dust temperature (Fig. 2; Crovisier et al. 2000).

Independent analyses of the 7 October spectrum were performed by Brucato et al. (1999), Colangeli et al. (1999a) and Wooden et al. (1999). The results are qualitatively similar, but different proportions of the silicate components are found, however. As discussed by Crovisier et al. (2000), this may be ascribed to the use of different laboratory samples, and to different assumptions on the silicate temperatures.

The 5-17 $\mu \mathrm{m}$ spectrum of comet P/Hartley 2 observed with CAM-CVF shows the 9-12 $\mu \mathrm{m}$ band of silicates (at a level of about $20 \%$ of the continuum, to be compared with $\approx 200 \%$ for comet Hale-Bopp) (Crovisier et al. 1999b, 2000). 


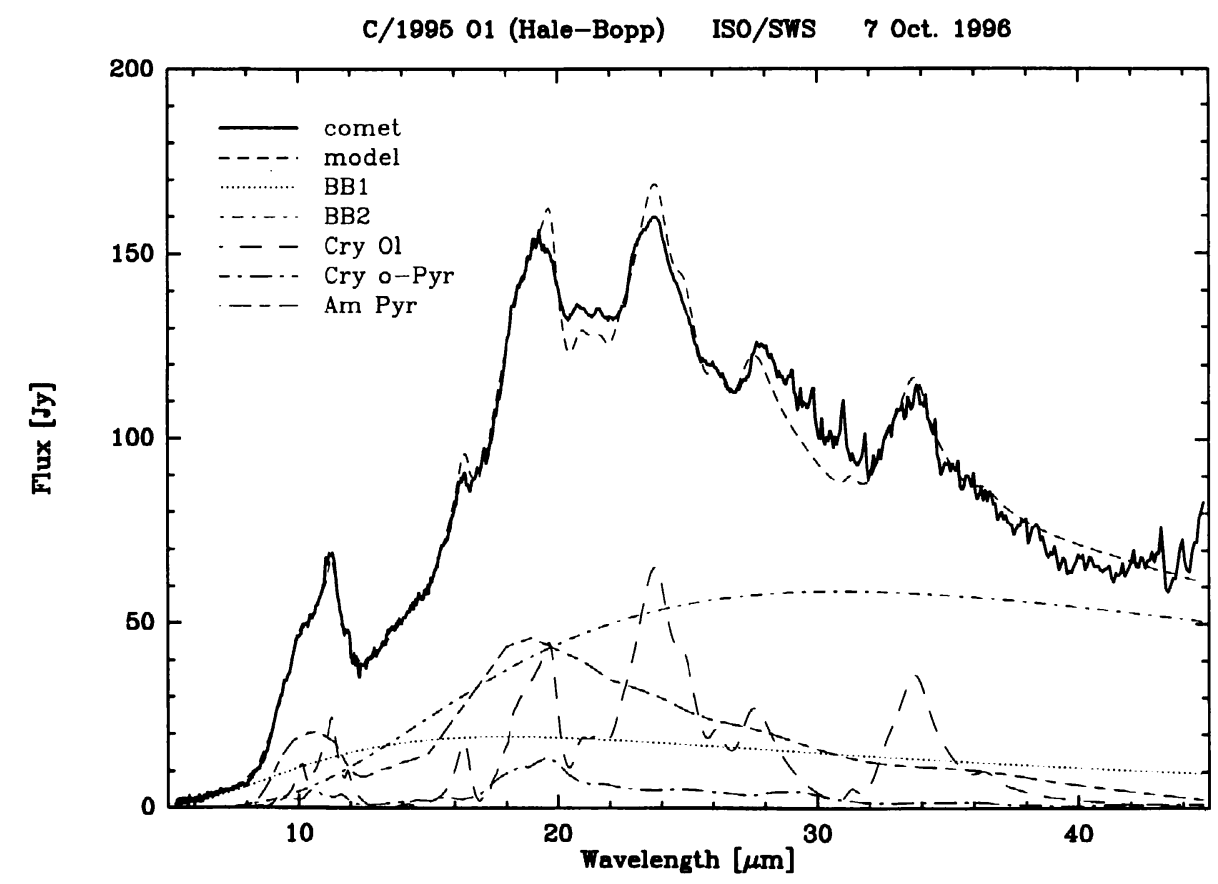

Figure 6. $\mathrm{C} / 1995 \mathrm{O} 1$ (Hale-Bopp) on 7 October 1996 at $r_{h}=2.8$ AU. The 6 to $45 \mu \mathrm{m}$ region of the SWS spectrum is represented here degraded to a resolution $\lambda / \Delta \lambda=500$ (full dark line). The intensity scale is normalized to a $14^{\prime \prime} \times 20^{\prime \prime}$ aperture assuming a $1 / \rho$ brightness distribution law. The observed spectrum has been decomposed into several emission components: two blackbody components, forsterite, ortho-pyroxene and amorphous silicates (from: Crovisier et al. 2000).

A possible emission at $11.3 \mu \mathrm{m}$ suggests the presence of crystalline silicates, which would be the first time it is found in a Jupiter-family comet.

No sign of PAHs could be found in these ISO spectra, especially in the 6-9 $\mu \mathrm{m}$ region where they have strong features unblended with silicates, which are conspicuous in many interstellar and circumstellar sources.

\section{Conclusion}

ISO observations of $\mathrm{C} / 1995 \mathrm{O} 1$ (Hale-Bopp) and 103P/Hartley 2 have revealed the spectra of comets over the full infrared range. These observations, complemented by ground-based infrared spectroscopy and by spectroscopy in other spectral domains, now allow us to have a comprehensive view of the composition of cometary volatiles, of the evolution of their production as a function of heliocentric distance, and of their physical conditions. A remarkable similarity with the abundances of interstellar molecules, in ices or in the gas phase, can 
be noted (cf. Bockelée-Morvan et al. 2000; Crovisier 1999; Irvine \& Bergin, this volume).

The thermal infrared spectrum of comet Hale-Bopp permitted the identification of several kinds of silicates. This spectrum closely resembles the spectra observed by ISO on the protoplanetary dust disks around some young stars (see Waelkens, this volume), emphasizing the similarity of the dust composition in both kinds of objects. This contrasts with interstellar silicates, which are primarily amorphous.

The history of cometary formation may be complex. Cometary nuclei may have retained unprocessed or little-processed matter from the primitive Solar Nebula, as well as processed matter such as crystalline silicates. Further investigations, especially by infrared spectroscopy, of comets from different families (i.e., of various origins) should help us in understanding their formation and the history of our Solar System.

Acknowledgments. This work is based on observations with ISO, an ESA project with instruments funded by ESA Member States (especially the PI countries: France, Germany, the Netherlands and the United Kingdom) and with participation of ISAS and NASA. I am grateful to the ISO teams for spectroscopic observations of comets (target-of-opportunity programme and central programme) and to L. Colangeli and his team, for sharing these results.

\section{References}

Bergin, E.A., Neufeld, D.A., Stauffer, J.R., et al. 1999, IAU Circ. No 7183

Bockelée-Morvan, D., Lis, D.C., Wink, D.E. , et al. 2000, A\&A, 353, 1101

Brucato, J.R., Colangeli, L., Mennella, V., Palumbo, P., \& Bussoletti, E. 1999, Planet. Space Scie., 47, 773

Colangeli, L., Brucato, J.R., Ferrini, L., et al. 1999a, Adv. Space Res., 23, 1243

Colangeli, L., Epifani, E., Brucato, J.R., et al. 1999b, A\&A, 343, L87

Crovisier, J. 1999, Earth Moon Planets, 79, 125

Crovisier, J., Brooke, T.Y., Hanner, M.S., et al. 1996, A\&A, 315, L385

Crovisier, J., Brooke, T.Y., Leech, K., et al. 2000, in Thermal Emission Spectroscopy and Analysis of Dust, Disks, and Regoliths, eds. M.L. Sitko, A.L. Sprague, \& D.K. Lynch, ASP Conf. Series, 196, in press

Crovisier, J., Encrenaz, T., Lellouch, E., et al. 1999b, ESA SP-427, 161

Crovisier, J., Leech, K., Bockelée-Morvan, D., et al. 1997a, Science, 275, 1904

Crovisier, J., Leech, K., Bockelée-Morvan, D., et al. 1997b, ESA SP-419, 137

Crovisier, J., Leech, K., Bockelée-Morvan, D., et al. 1999a, ESA SP-427, 137

Kessler, M.F., Steinz, J.A., Anderegg, M.E., et al. 1996, A\&A, 315, L27

Lellouch, E., Crovisier, J., Lim, T., et al. 1998, A\&A, 339, L9

Mumma, M.J., Weissman, P.R., \& Stern, S.A. 1993, in Protostars and Planets

III, eds. E.H. Levy \& J.I. Lunine, Univ. Arizona Press, 1177

Wooden, D.H., Harker, D.E., Woodward, C.E., et al. 1999, ApJ, 517, 1034 


\section{Discussion}

T. Millar: If $\mathrm{H}_{2} \mathrm{O}$ sublimes through a liquid phase, as suggested by Bill Irvine in his talk, could this affect the ortho-para ratio?

J. Crovisier: This is clearly the kind of processes which should be investigated in the laboratory.

J. Storey: Why are PAH's so much less evident in cometary spectra than in the interstellar medium?

J. Crovisier: One reason could be that PAH's are not abundant in cometary material. Another reason, for the Hale-Bopp ISO spectra, is that they were observed at heliocentric distances $>2.8 \mathrm{AU}$, where the temperature of cometary grains might be too low to allow the release of PAH's in the coma. It must be noted that some (but not all) comets show a band at $3.28 \mu \mathrm{m}$ indicative of the presence of aromatics. 